\title{
Behavioural Study of Captive Sloth Bears Using Environmental Enrichment Tools
}

\author{
M. Veeraselvam, ${ }^{1,2}$ R. Sridhar, ${ }^{1,3}$ M. G. Jayathangaraj, ${ }^{1}$ and P. Perumal ${ }^{4}$ \\ ${ }^{1}$ Department of Wildlife Science, Madras Veterinary College, Tamil Nadu Veterinary and Animal Sciences University, \\ Chennai 600 051, India \\ ${ }^{2}$ Veterinary University Training and Research Centre, TANUVAS, Salem 636001, India \\ ${ }^{3}$ Department of Veterinary Pathology, Madras Veterinary College, TANUVAS, Chennai 600007, India \\ ${ }^{4}$ National Research Center on Mithun, ICAR, Jharnapani, Nagaland 797106, India
}

Correspondence should be addressed to M. Veeraselvam; drveeraselva@gmail.com

Received 31 August 2013; Accepted 18 November 2013

Academic Editor: Michel Laurin

Copyright (c) 2013 M. Veeraselvam et al. This is an open access article distributed under the Creative Commons Attribution License, which permits unrestricted use, distribution, and reproduction in any medium, provided the original work is properly cited.

\begin{abstract}
Effects of environmental enrichment on behavioural changes were studied in five captive sloth bears kept in confined enclosure at Zoological Park, Chennai, India. Behavioural categories like active, passive, and abnormal behaviours were taken for the study. The activity budget was recorded as a single animal scan. The detailed baseline data of 150 hours, over a period of 30 days, were collected. At baseline, bears exhibited passive and more abnormal behaviours. Similarly, after application of the environmental tools like honey-log, underground food pipes, and wobbling box in the enclosure, the data were collected for 150 hours ( 30 days). Increased active behaviours and decreased abnormal behaviours were observed and showed highly significant changes in the abnormal behaviour as a whole when compared to the baseline level. During the postenrichment period, the data that were collected for 150 hours (30 days) showed no significant differences statistically between the behavioural categories. But certain level of difference was evident from the percentage of abnormal behaviours exhibited by individual bears. Among the enrichment devices, honey-log was the most preferred enrichment tool as revealed by the percentage of time spent by individual animal. The results show that application of enrichment tool continuously may bring long term effect in stereotypic behaviour.
\end{abstract}

\section{Introduction}

Bears are large, impressive, extremely popular zoo animals and the subject of considerable folklore [1]. Among them sloth bears (Melursus ursinus) are widely distributed on the Indian subcontinent and on the Islands of Sri Lanka [2]. Zoos have responsibility to provide the best possible house for animals living in restricted environments [3]. The purpose may be to increase the natural habitat, to increase behavioural diversity and number of normal behavioural patterns, to reduce the frequencies of abnormal behavior, to increase reproductive success, or to make them more visible to the public $[4,5]$.

Most of the bear species kept under captivity exhibited abnormal behaviours because of the small enclosures in the zoos [6]. This greatly reduced the opportunity to exhibit its natural behaviour [7]. Bears kept in such barren environments show an overall decrease in interaction with the environment that tends to perform stereotypies [8]. Stereotypies are high repetitive uniform sequences of behaviour, which seem to be of no functional value to the animals [9]. However, the barren environments are not the unique reason for stereotypies. Age, husbandry procedures [10], rearing history, [1] and genetic factor [11] have been implicated in the cause of stereotypic behaviours. Since welfare and occupational engagement of animals are of utmost importance for the successful conservation and breeding of animals modern day zoos are implementing environmental enrichment.

Environmental enrichment aims at stimulating a wide range of species-specific behaviours, which are performed in normal sequences and frequencies. The use of environmental enrichment simultaneously reduces the stereotypic and abnormal repetitive behaviours and enhances the wellbeing of zoo animals [12]. To alleviate captivity induced stereotypism various zoos around the world are considering environmental enrichment as a tool in their management 
protocol and often consist in new feeding methods [8, 13-15] or in new facilities $[16,17]$.

Several studies demonstrated that variability of the environment contributed to enrichment by stimulating natural behaviour and preventing animals from abnormal behaviour. Carlstead et al. [6] reported that honey-filled wooden logs helped to stimulate investigatory behaviour and exploration and reduced the stereotypy pacing in sloth bears (Melursus ursinus), American black bears (Ursus americanus), and brown bears (Ursus arctos). Forthman and Bakeman [1] observed that novel feeding enrichment like ice blocks containing fishcicles and various layers of scattered peanuts, bread, raisins, peanut butter, and sunflower seeds had a significant positive influence on the behaviour of captive bears. Gupta et al. [15] used various types of enrichment tools like scented log, elevated platforms, betting/nesting materials, scattering of feed, and water ponds on rotational basis to sloth bears to mimic natural food scavenging activity and making the animal content. In the present study sloth bears were considered for their welfare related worthiness in zoo exhibits, by the way of supplementing various enrichment protocols, specifically suited for them to encourage natural behavioural repertoire common in wilderness. The objective of this study is to determine the effectiveness of environmental enrichment with various types of models on the behavioural activity pattern of sloth bear.

\section{Material and Methods}

2.1. Place of Study. The behavioural study on captive sloth bears was carried out at Arignar Anna Zoological Park, which is located on the Eastern side of the Grand Southern Trunk road (NH 45), South of Chennai city. It is one of the biggest zoos in Southeast Asia extending over 510 ha. The zoo is open to the public from 09:00 to 17:00 hrs of the day.

2.2. Subjects. Observation was carried out on sloth bears (1 male and 4 female) displayed in Arignar Anna Zoological Park, Vandalur, Chennai, India. The bears were as follows: Raja (male), Pattu (female), Geetha (female), Banu (female), and Rani (female). The ages were approximated based on the records which claim it to be between 4 and 15 years. The animals were rescued from circuses and poachers. The bears were housed in separate cages and were released into the enclosure (day crawl) each morning. The routine activities included delivery of the daily feeding in the cages which was between 12:00 and 13:00 hrs of the day. Bears entered their respective cages voluntarily for the routine feeding without any infighting.

2.3. Exhibit Area. During daylight, the sloth bears (both male and females) were kept together in the enclosure, consisting of a rock wall (10 meters) on the south side and 6-meter-deep moat on the other side that separated the animals from the visitors. A water pool for drinking was maintained at the centre of the day crawl. The enclosure was furnished with natural soil and living vegetation. There was no other manipulable furnishing in the enclosure prior to the application of enrichment devices.
2.4. Experimental Treatments. A major part of the natural behaviour repertoire of sloth bears consists of foraging and food handling activities. By increasing the opportunities for sloth bears at the Arignar Anna Zoological Park to forage, it was predicted that their explorative and manipulative behaviour would be stimulated, consequently challenging the animals on a cognitive level. The enrichment treatments tested were the following.

2.4.1. Honey-Log. A hollow bamboo $\log 30 \mathrm{~cm}$ long and $10 \mathrm{~cm}$ in diameter was cut at the internodes. This cylindrical bamboo closed at both ends was made available with one hole of $1 \mathrm{~cm}$ in diameter drilled into the center of each internode. This was slightly modified from the methods suggested by Carlstead et al. [6]. Before presentation to the bear on each morning, the log was filled with approximately $200-250 \mathrm{~g}$ of honey with the help of a funnel. It was firmly tied to a tree in such a way that the bear could just reach it to suck the honey. It was placed for 10 consecutive days.

2.4.2. Underground Food Pipes. Law et al. [18] used underground food pipes at Glasgow zoo for black bears quoted in Shepherdson [3]. Based on this model, a modified method was adopted. Four polyvinyl chloride (PVC) pipes of about 6 inches in length each and a diameter of 1 inch were cast in a square concrete block of one and half feet by one foot and a thickness of 6 inches. The pipes were placed in four corners leaving one end of the pipe free for exploration in the concrete block. The concrete block was firmly buried in level with the ground leaving the top layer accessible. Cut fruits, raisins, dry figs, and peanuts were placed inside the pipes through the open end daily for a period of ten consecutive days.

2.4.3. Wobbling Box. A plywood box of 1 foot by 1 foot was selected. The top portion of the box was removed. The remaining four sides were drilled with two rows of holes 2 inches in diameter leaving enough space between holes. The bottom plank was anchored to an 8-12-feet wooden pole (6 inches in diameter) placed vertically from the ground. Various treats like cut fruits, raisins, and nuts were placed in the box through the uncovered portion. Care was taken not to anchor the wooden pole tightly to the ground. The primary objective was to provide easy manipulation and shaking which rewarded the bears with treats falling out through the drilled holes. They were refilled every day for a period of 10 consecutive days.

2.5. Data Collection. Preliminary observations were conducted on all the five bears in the enclosures to standardize the behavioural pattern exhibited and to develop an ethogram (Table 1) modified from Forthman and Bakeman [1] and Renner and Lussier [17]. The group of five bears exhibited between 09:00 and 17:00 hrs daily were studied. The activity budget was recorded as a single animal scan [19]. On the basis of these observations, the main study began on the 3rd of November, 2006, and ran until the 28th of February, 2007. Behavioural categories like active, passive, and abnormal behaviours were studied excluding the period when the bears 
TABLE 1: Ethogram.

\begin{tabular}{|c|c|}
\hline \multicolumn{2}{|r|}{ Active behaviour } \\
\hline Exploring & $\begin{array}{l}\text { Searching for things inside its enclosure like dead branches, stones, walls, cages, artificial objects, } \\
\text { and so forth }\end{array}$ \\
\hline Locomotion & Moving from one place to another inside its enclosure quadrupedally or bipedally \\
\hline Fighting & Quarrelling with its counterparts \\
\hline Climbing & Moving up the tree trunk \\
\hline Digging & Making a hole on the ground with its paws \\
\hline Rolling & Turning itself on the ground \\
\hline Playing & Playing and wrestling with inhibited strikes with low or no vocalization \\
\hline Social & Playing with its other enclosure mates in a friendly way \\
\hline Solitary & Playing all by itself with inanimate objects inside its enclosure \\
\hline Foraging & Manipulating food items; this includes live plant material and drinking of water \\
\hline Object observation & Perusing the inanimate objects in its enclosure like logs, rocks, and walls \\
\hline \multicolumn{2}{|r|}{ Passive behaviour } \\
\hline Rest & Bear sits or lies with its head down or eyes visibly closed \\
\hline Alert & Bear lies, sits, or stands with head up and eyes open and responds to any stimuli \\
\hline Maintenance & Bear grooms itself with its mouth and paws, scratches, defecates, urinates, and rubs its body \\
\hline \multicolumn{2}{|r|}{ Abnormal behaviour } \\
\hline Pacing & Bear traverses the same path repetitively \\
\hline Swaying & Moving its body to and fro by standing \\
\hline Head tossing & Moving the head up and down \\
\hline Self-aggression & Action directed towards other bears \\
\hline Circling & Moving in a circular direction constantly \\
\hline Self-mutilation & Biting its own skin repeatedly to the extent of inducing trauma \\
\hline
\end{tabular}

Modified from Forthman et al. [8] and Renner and Lussier [17].

could not be seen. Since the sample size was small and for ease of work, all stereotypic behaviours were considered under abnormal behaviours.

2.6. Baseline Activity Budget. Baseline behavioural data on all the five bears were collected using a scan sampling method $[19,20]$. Two 3-hour segments [21] in each day were collected, respectively; 09:00-12:00 and 13:00-16:00 hrs segment times were selected to encompass daylight hours and accommodate feeding. Data were collected in the above segmented periods with each animal's behaviour recorded once per minute per animal [17] for 30 hours with a 1-minute interval for every cycle (5 minutes) of observation of the five bears. A total of $150 \mathrm{hrs}$ of baseline data were collected for 30 days.

2.7. Enrichment Activity Budget. Following the baseline data collection, enrichment devices were installed. Each morning, before the bears were sent out, the enrichment devices were placed in the exhibit area according to the experimental condition. Each device, namely, honey-log, underground food pipes, and wobbling box, was installed for 10 consecutive days in various locations. In this enrichment period the data were collected for 150 hours (30 days), using the same method applied for baseline data collection. Enrichment materials were removed at the end of the day from the day crawl to facilitate refilling.
2.8. Postenrichment Activity Budget. Postenrichment data were collected after removing all the enrichment tools from the enclosures. The data were collected for $150 \mathrm{hrs}$ (30 days) using the same method applied for enrichment data collection.

2.9. Statistical Analysis. Differences among times spent in behavioural categories of baseline, enrichment and postenrichment and differences among the times utilized on each enrichment tool were evaluated by ANOVA as per the standard procedures given by Snedecor and Cochran [22]. A value of $P<0.05$ was considered significant.

\section{Results}

3.1. Effect of Environmental Enrichment on Behavioural Categories. The hours of time spent in each behavioural category (active, passive, and abnormal) during baseline period were compared to the effects of enrichment and postenrichment period.

3.2. Active Behaviour. Active behaviour states were studied in a detailed ethogram constituting the baseline data which was compared after supplementation of enrichment devices and the withdrawal of the same. The mean \pm SE values for the baseline, enrichment, and postenrichment data were 
TABLE 2: Effect of environmental enrichment on behavioural categories.

\begin{tabular}{lccr}
\hline \multirow{2}{*}{ Source } & \multicolumn{1}{c}{} & Mean \pm SE values & Abnormal \\
& Active & Passive & $16.43 \pm 1.85^{\mathrm{a}}$ \\
\hline Baseline & $11.27 \pm 1.83^{\mathrm{a}}$ & $2.29 \pm 0.16$ & $1.21 \pm 0.37$ \\
Enrichment & $23.94 \pm 0.61^{\mathrm{b}}$ & $1.85 \pm 0.37$ & $4.21 \pm 0.42^{\mathrm{b}}$ \\
After enrichment & $12.43 \pm 1.79^{\mathrm{a}}$ & $2.40 \pm 0.28$ & $15.17 \pm 1.74^{\mathrm{a}}$ \\
\hline
\end{tabular}

Within columns means with different letters (a, b) differ significantly $(P<0.01)$.

TABLE 3: Activity budget with enrichment tools.

\begin{tabular}{lc}
\hline Treatment & Mean \pm SE value \\
\hline Honey-log & $7.64 \pm 1.01$ \\
Underground food pipes & $6.13 \pm 0.64$ \\
Wobbling boxes & $4.44 \pm 0.81$ \\
\hline
\end{tabular}

NS: nonsignificant.

$11.27 \pm 1.82,23.94 \pm 0.61$, and $12.43 \pm 1.79$, respectively. From the above results, there was a highly significant variation $(P<$ 0.01 ) in enrichment study as compared to the baseline and postenrichment studies (Table 2).

3.3. Passive Behaviour. The baseline, enrichment, and postenrichment studies for passive behaviours were carried out. The mean \pm SE values for the abovementioned behavioural categories were estimated to be $2.29 \pm 0.16,1.85 \pm 0.37$, and $2.40 \pm 0.28$, respectively. As compared to the baseline study the percentage of passive behaviour was lowered in the enrichment and postenrichment studies. However, there were no significant variations in the mean value of the baseline, enrichment, and postenrichment studies (Table 2).

3.4. Abnormal Behaviour. The mean \pm SE values for the baseline, enrichment, and after enrichment were estimated to be $16.43 \pm 1.85,4.21 \pm 0.42$, and $15.17 \pm 1.74$, respectively. The mean \pm SE values of the enrichment data showed highly significant variation $(P<0.01)$ in comparison with the baseline and postenrichment data (Table 2).

3.5. Activity Budget with Enrichment Tools. Enrichment tools comprising honey-log, underground food pipes, and wobbling box were used on a rotational basis for a period of 10 days for each enrichment tool, constituting a total of 150 hours (30 days). Out of the 150 hours, a total of 91 hours were utilized on these tools cumulatively by all the 5 bears with greater time spent on honey-log ( $35.20 \mathrm{hrs})$ than underground food pipes (30.25 hrs) and wobbling box (26.00 hrs). The mean \pm SE values were estimated for honey-log, underground food pipes, and wobbling box for their differences in preference and usage. Mean $\pm \mathrm{SE}$ values were estimated to be $7.64 \pm 1.01,6.13 \pm 0.64$, and $4.44 \pm 0.81$, respectively. No significant variations were noticed on the preference and usage of these enrichment tools (Table 3).

\section{Discussion}

4.1. Effect of Environmental Enrichment on Behavioural Categories. The time activity budget of the captive sloth bears in this study consisted of active, passive, and abnormal behaviours. In the present study, detailed baseline data was collected for a period of $150 \mathrm{hrs}$ distributed across 30 days. Sloth bears studied were less active and passive and exhibited more abnormal stereotypic behaviours. This is in accordance with the finding proposed by Carlstead and Seidensticker [13] who opined that the stereotypies and abnormal behaviours were common among confined bears.

In this study, application of environmental enrichment revealed highly significant differences in the various behavioural categories of sloth bears when compared to baseline levels. This is similar to the finding reported by Renner and Lussier [17] that the use of environmental enrichment brought highly significant changes in the various behavioural categories. Here, bears showed an overall increase in time spent active, decrease in time spent passive, and less engagement in abnormal behaviours.

4.2. Active Behaviour. Environmental enrichment greatly increased the time spent active by bears in the zoo enclosure. This was also further evident by the differences in percentage of activity exhibited by individual bears when compared with their respective baseline activities.

All the five bears ( 1 male and 4 female) showed overall increase in their activity probably due to the introduction of novel enrichment tools in the enclosure. The difference was noticed with the bears spending more time on exploring, foraging, climbing, and digging which was in agreement with Carlstead et al. [6] who reported similar activities after enrichment.

Similarly, the active behaviour after enrichment showed no significant differences when compared with baseline levels and enrichment period probably as a result of the short experimental duration. Fischbacher and Schmid [14] opined that a long term environmental protocol was needed to bring about changes in general activity and welfare of the animals.

4.3. Passive Behaviour. In this study, passive behaviours reduced when compared to the baseline activity but it was not statistically significant as reported by Atten [23]. The same level of passive behaviour was observed during the postenrichment period probably because the short duration of study limits the opportunity for habituation to take place as reported by Swaisgood et al. [24]. 
4.4. Abnormal Behaviours. All the five bears studied exhibited abnormal behaviours (stereotypic) like pacing, swaying, head tossing, circling, and self-aggression. This finding was in accordance with Vickery and Mason [10] who opined that stereotypic behaviours were very common in Ursids kept under captivity.

All the animals irrespective of sex exhibited stereotypic pacing which revealed sex does not influence pacing behaviour. Moreover, during the baseline data collection, stereotypic pacing showed a maximum predominance in the stereotypic behavioural repertoire of the animals. Further, all the bears studied were rescued from gypsies where they had a history of being tied constantly to a tree or a pole. Hence, rearing history, boredom, and lack of occupation could have influenced the animal to exhibit abnormal behaviours. This was strongly supported by Forthman and Bakeman [1] who reported that rearing history was found to strongly influence stereotypic pacing, head swaying, circling, and selfaggression in captive sloth bears.

With the introduction of enrichment tools, all the bears showed a change in exhibiting natural behavioural activities. The enrichment though provided for a short period brought about highly significant changes in the abnormal behaviour as a whole when compared to the baseline level. This was in accordance with the finding of Renner and Lussier [17] who reported that the provision of various enrichment tools like climbing structure and ice cubes with fishcicles in the enclosure brought about a significant reduction in stereotypic behaviour. Though animals were found very active during enrichment schedule, food anticipation behaviour was evident just before feeding which was in agreement with Montaudouin and Le Pape [25] and Vickery and Mason [10] who observed similar changes.

Similarly, comparison between baseline levels and postenrichment levels revealed no significant difference statistically. But certain levels of difference were evident from the percentage of abnormal behaviours exhibited by individual bears. This was supported by Altmann [19] who reported that during postenrichment period there was a slight increase in abnormal and passive behaviours and a decrease in overall activity. He concluded that any short term enrichment protocol will not bring about any long lasting effects in curtailing stereotypism. In the present enrichment study because of the time constraints a total of only 30 days were chosen. Probably an extended experimental schedule could have provided better results.

4.5. Activity with Enrichment Tools. In the present study, three novel objects, honey-log, underground food pipes, and the wobbling box, were used. These items were carefully chosen and modified in such a way that they provided an opportunity for the sloth bear to express those behaviours that occur naturally in the wild which was in agreement with Swaisgood et al. [24] when they selected the enrichment tools for pandas. These three items were placed in the enclosure for a period of 10 days each and no significant difference was noticed except that the order of preference was more towards the honey-log as it was the most preferred food by sloth bears as also reported by Laurie and Seidensticker [26].
Though honey was the most preferred food the modified presentation of the honey-log could have played a role here. Honey-log with a hole drilled on either side was presented here at a reasonable height and finally tied parallel to the ground. This increased the complexity of accessing the honey as the log had to be turned vertically for the honey to flow out. Hence, more time was spent with it than if it had been presented in a vertical direction. Reasons for the increased time spent were probably attributed to the increased complexity, competition, reduced access, and increased occupational involvement. This finding was further supported by Carlstead and Seidensticker [13] who reported that any effective presentation of the enrichment device increased the competition. Investigation and occupation thereby were reducing stereotypies in sloth bears.

Similarly, underground food pipes (PVC pipes) cast in a square concrete block placed underground exposing the open end were developed to stimulate natural sucking of termites by sloth bears [26]. Hence, instead of termites, treats like cut fruits, raisins, dry figs, and peanuts were placed inside the pipes which were sucked out. Law et al. [18] successfully used this novelty at Glasgow zoo for black bears to increase digging activity cited in Shepherdson [3].

Wobbling box was also a novel concept tried for the first time for behavioural study purpose. In this enrichment model, the holes were drilled only on the four sides of the box and it was deliberately avoided in the bottom of the plank for the treats to remain in the box for a longer duration. Here, the sloth bears were found standing on their hind feet and manipulating the device. Their repeated shaking of the box made the treats scattered all around. This behaviour was common in the wild in food manipulation of trees and searching for the objects fallen down. Grandia et al. [27] opined that scattered feeding increased active and foraging behavioural categories in bears.

In this study, the environmental enrichment tools were effective in eliciting naturalistic behaviours among all the five sloth bears. This concludes that application of enrichment tool continuously may bring long term effect in stereotypic behaviour.

\section{Conflict of Interests}

The authors declare that there is no conflict of interests regarding the publication of this paper.

\section{Acknowledgments}

The authors gratefully acknowledge the support from Sri. Sukdev, Principal Chief Conservator of Forest, Tamil Nadu, and Mr. Anandhaswamy, Director and Chief Conservator of Forest, Arignar Anna Zoological Park, Chennai, India.

\section{References}

[1] D. L. Forthman and R. Bakeman, "Environmental and social influences on enclosure use and activity patterns of captive sloth bears (Ursus ursinus)," Zoo Biology, vol. 11, pp. 405-415, 1992. 
[2] D. L. Prater, The Book of Indian Mammals, Oxford University Press, Bombay, India, 3rd edition, 1965, Bombay Natural History Society.

[3] D. Shepherdson, "A wild time at the zoo: practical enrichment for zoo animals," Zoo Zen, vol. 8, no. 5, pp. 1-9, 1992.

[4] R. J. Young, Environmental Enrichment for Captive Animals, Blackwell Science, 2003.

[5] S. Montaudouin and G. Le Pape, "Comparison between 28 zoological parks: stereotypic and social behaviours of captive brown bears (Ursus arctos)," Applied Animal Behaviour Science, vol. 92, no. 1-2, pp. 129-141, 2005.

[6] K. Carlstead, J. Seidensticker, and R. Baldwin, "Environmental enrichment for zoo bears," Zoo Biology, vol. 10, pp. 3-16, 1991.

[7] R. R. Swaisgood and D. J. Shepherdson, "Scientific approaches to enrichment and stereotypies in zoo animals: what's been done and where should we go next?" Zoo Biology, vol. 24, no. 6, pp. 499-518, 2005.

[8] D. L. Forthman, S. D. Elder, R. Bakeman, T. W. Kurkowski, C. C. Noble, and S. W. Winslow, "Effects of feeding enrichment on behaviour of three species of captive bears," Zoo Biology, vol. 11, pp. 187-195, 1992.

[9] G. J. Mason, "Stereotypies: a critical review," Animal Behaviour, vol. 41, no. 6, pp. 1015-1037, 1991.

[10] S. Vickery and G. Mason, "Stereotypic behavior in Asiatic black and Malayan sun bears," Zoo Biology, vol. 23, no. 5, pp. 409-430, 2004.

[11] D. Liu, Z. Wang, H. Tian et al., "Behavior of giant pandas (Ailuropoda melanoleuca) in captive conditions: gender differences and enclosure effects," Zoo Biology, vol. 22, no. 1, pp. 77-82, 2003.

[12] G. Mason, R. Clubb, N. Latham, and S. Vickery, "Why and how should we use environmental enrichment to tackle stereotypic behaviour?" Applied Animal Behaviour Science, vol. 102, no. 3-4, pp. 163-188, 2007.

[13] K. Carlstead and J. Seidensticker, "Seasonal variation in stereotypic pacing in an American black bear Ursus americanus," Behavioural Processes, vol. 25, no. 2-3, pp. 155-161, 1991.

[14] M. Fischbacher and H. Schmid, "Feeding enrichment and stereotypic behavior in spectacled bears," Zoo Biology, vol. 18, no. 5, pp. 363-371, 1999.

[15] B. K. Gupta, A. K. Sinha, and S. Prakash, "Effect of environmental enrichment on behaviour of sloth bears (Melursus ursinus)," Current Science, vol. 86, no. 3, pp. 389-391, 2004.

[16] M. J. Renner, A. J. Feiner, M. G. Orr, and B. A. Delaney, "Environmental enrichment for new world primates: introducing food relevant objects and direct and secondary effects," Journal of Applied Animal Welfare Science, vol. 3, no. 1, pp. 23-32, 2000.

[17] M. J. Renner and J. P. Lussier, "Environmental enrichment for the captive spectacled bear (Tremarctos ornatus)," Pharmacology Biochemistry and Behavior, vol. 73, no. 1, pp. 279-283, 2002.

[18] G. Law, H. Boyle, J. Johnson, and A. Macdonald, "Food presentation. Part 1-Bears," Ratel, vol. 17, no. 2, pp. 44-47, 1990.

[19] J. Altmann, "Observational study of behavior: sampling methods," Behaviour, vol. 49, no. 3-4, pp. 227-267, 1974.

[20] P. Martin and P. Bateson, Measuring Behaviour: An Introductory Guide, Cambridge University Press, Cambridge, UK, 2nd edition, 1994.

[21] N. J. Ley, Time budget analysis of asiatic black bears with a focus on stereotypic behaviour [M.S. thesis], Wild Animal Biology, University of London, 2004.

[22] G. W. Snedecor and W. G. Cochran, Statistical Methods, Iowa State University, Ames, Iowa, USA, 8th edition, 1967.
[23] L. E. V. Atten, Using environmental enrichment as an effective tool for promoting natural behavioural in blind and fully-sighted sloth bears in captivity [M.S. thesis], Applied Animal Behaviour and Animal Welfare at the University of Edinburgh, 2006.

[24] R. R. Swaisgood, A. M. White, X. Zhou et al., "A quantitative assessment of the efficacy of an environmental enrichment programme for giant pandas," Animal Behaviour, vol. 61, no. 2, pp. 447-457, 2001.

[25] S. Montaudouin and G. Le Pape, "Comparison of the behaviour of European brown bears (Ursus arctos arctos) in six different parks, with particular attention to stereotypies," Behavioural processes, vol. 67, no. 2, pp. 235-244, 2004.

[26] A. Laurie and J. Seidensticker, "Behavioural ecology of the sloth bear (Melursus ursinus)," Journal of Zoology, vol. 182, pp. 187204, 1977.

[27] P. A. Grandia, J. J. V. Dijk, and P. Koene, "Stimulating natural behavior in captive bears," Ursus, vol. 12, pp. 199-202, 2000. 

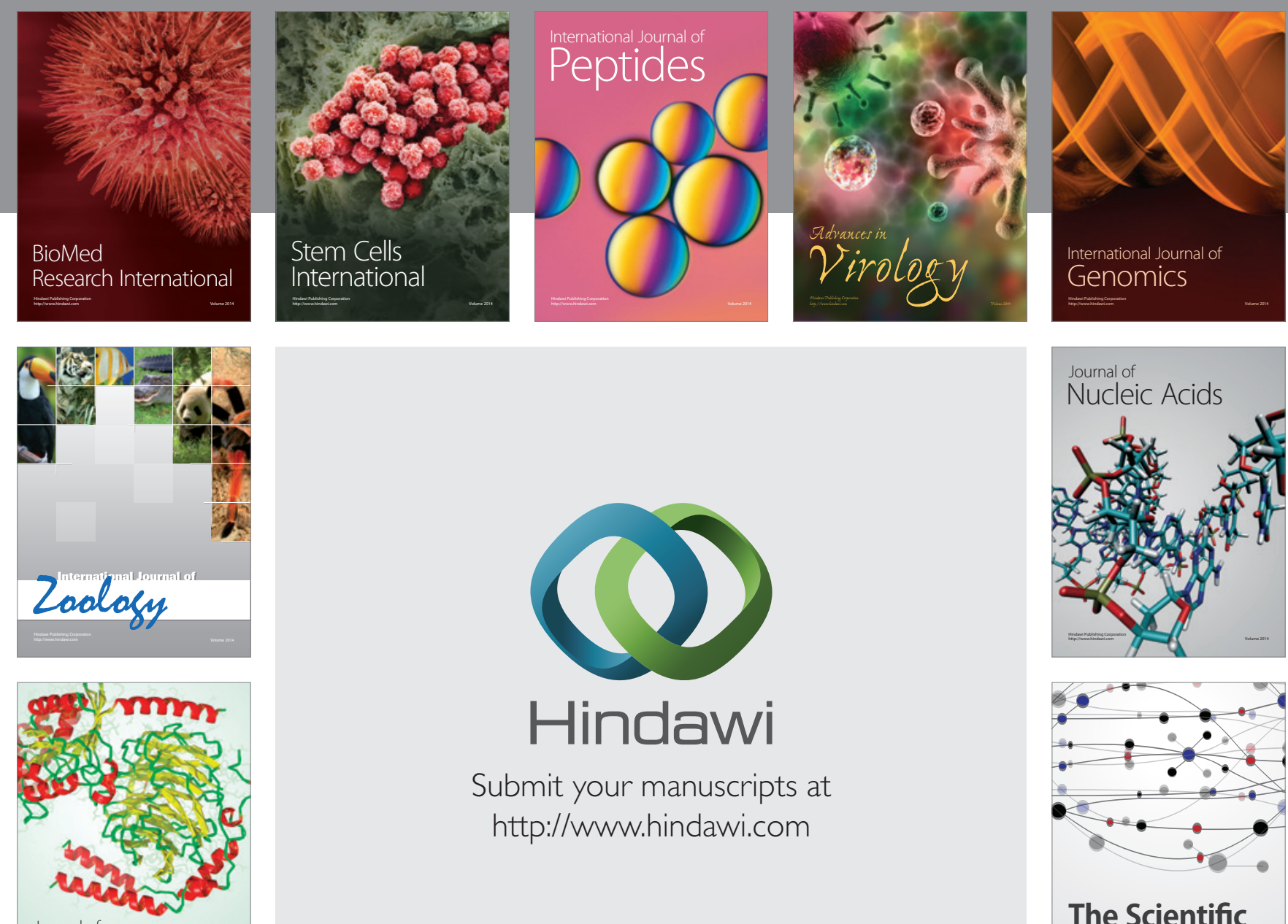

Submit your manuscripts at

http://www.hindawi.com

Journal of
Signal Transduction
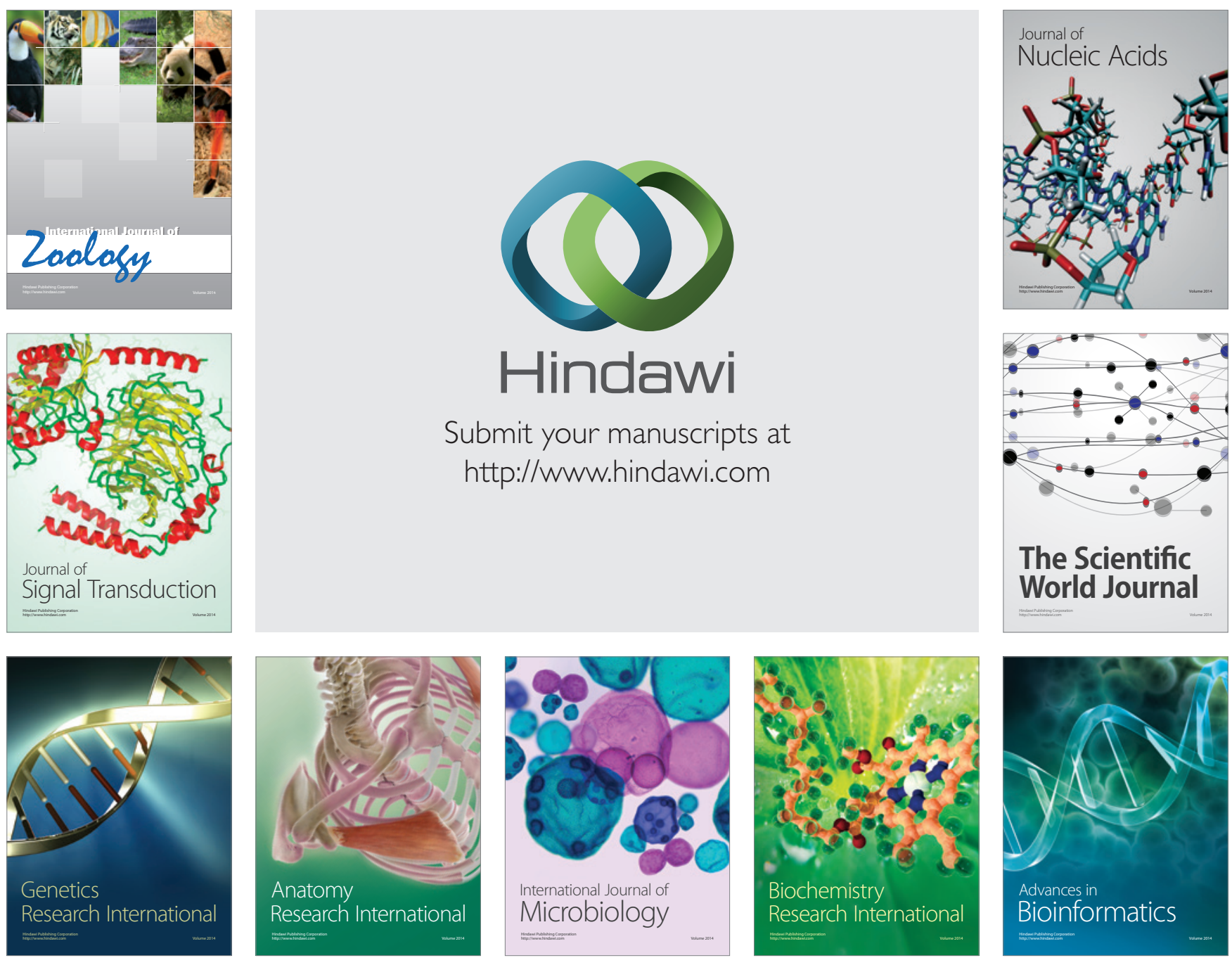

The Scientific World Journal
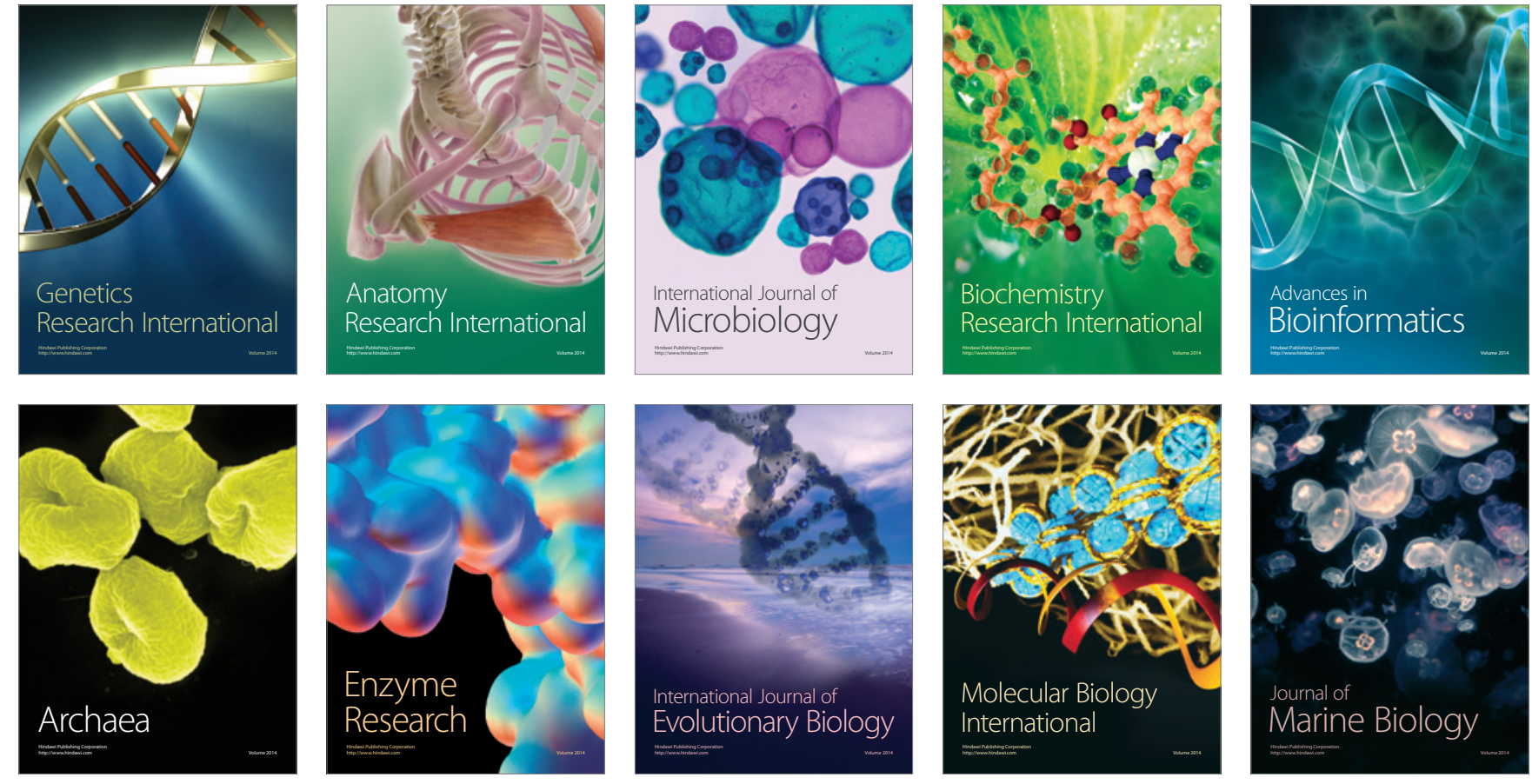